\title{
Stateless Multicasting in Mobile Ad Hoc Networks
}

\author{
Xiaojing Xiang, Member, IEEE, Xin Wang, Member, IEEE, and Yuanyuan Yang, Fellow, IEEE
}

\begin{abstract}
There are increasing interest and big challenge in designing a scalable and robust multicast routing protocol in a mobile ad hoc network (MANET) due to the difficulty in group membership management, multicast packet forwarding and the maintenance of multicast structure over the dynamic network topology for a large group size or network size. In this paper, we propose a novel Robust and Scalable Geographic Multicast Protocol (RSGM). Several virtual architectures are used in the protocol without need of maintaining state information for more robust and scalable membership management and packet forwarding in the presence of high network dynamics due to unstable wireless channels and node movements. Specifically, scalable and efficient group membership management is performed through a virtual-zone-based structure, and the location service for group members is integrated with the membership management. Both the control messages and data packets are forwarded along efficient tree-like paths, but there is no need to explicitly create and actively maintain a tree structure. The stateless virtual-tree-based structures significantly reduce the tree management overhead, support more efficient transmissions, and make the transmissions much more robust to dynamics. Geographic forwarding is used to achieve further scalability and robustness. To avoid periodic flooding of the source information throughout the network, an efficient source tracking mechanism is designed. Furthermore, we handle the empty zone problem faced by most zone-based routing protocols. We have studied the protocol performance by performing both quantitative analysis and extensive simulations. Our results demonstrate that RSGM can scale to a large group size and a large network size, and can more efficiently support multiple multicast groups in the network. Compared to existing protocols ODMRP and SPBM, RSGM achieves a significantly higher delivery ratio under all circumstances, with different moving speeds, node densities, group sizes, number of groups and network sizes. RSGM also has the minimum control overhead and joining delay.
\end{abstract}

Index Terms-Multicast routing, geographic multicast, mobile computing, wireless networks, mobile ad hoc networks, geographic routing, location, scalable, robust.

\section{INTRODUCTION}

There are increasing interests and use of mobile ad hoc networks with the fast progress of computing techniques and wireless networking techniques. In a mobile ad-hoc network (MANET), wireless devices could self-configure and form a network with an arbitrary topology. The network's topology may change rapidly and unpredictably. Such a network may operate in a standalone fashion, or may be connected to the larger Internet. Mobile ad-hoc networks became a popular subject for research in recent years, and various studies have been made to increase the performance of ad hoc networks and support more advanced mobile computing and applications [1], [2], [3].

X. Xiang is currently with Microsoft Corporation, Redmond, Washington 98052, USA. X. Wang and Y. Yang are with Stony Brook University, Stony Brook, New York 11794, USA.
Multicast is a fundamental service for supporting information exchanges and collaborative task execution among a group of users and enabling cluster-based computer system design in a distributed environment. Although it is important to support multicast in a mobile ad hoc network (MANET), which is often required by military and emergency applications, there is a big challenge to design a reliable and scalable multicast routing protocol in the presence of frequent topology changes and channel dynamics.

Many efforts have been made to develop multicast protocols for MANETs. These include conventional tree-based protocols and mesh-based protocols. The tree-based protocols (e.g., LAM [13], MAODV [19], AMRIS [24], MZRP [25]) construct a tree structure for more efficient multicast packet delivery, and the tree structure is known for its efficiency in utilizing network resources. However, it is very difficult to maintain the tree structure in mobile ad hoc networks, and the tree connection is easy to break and the transmission is not reliable. The mesh-based protocols (e.g., FGMP [4], Core-Assisted Mesh protocol [11], ODMRP [12]) are proposed to enhance the robustness with the use of redundant paths between the source and the set of multicast group members, which incurs a higher forwarding overhead. There is a big challenge to support reliable and scalable multicast in a MANET with these topology-based schemes, as it is difficult to manage group membership, find and maintain multicast paths with constant network topology changes.

In order to support more reliable and scalable communications, it is critical to reduce the states to be maintained by the network, and make the routing not significantly impacted by topology changes. Recently, several location-based multicast protocols have been proposed [16], [17], [18] for MANET. These protocols assume mobile nodes are aware of their own positions through certain positioning system (e.g., GPS), and make use of geographic routing to transmit packets along the multicast trees. In these protocols, a multicast packet carries the information of the entire tree or all the destinations into the packet headers, thus there is no need to distribute the routing states in the network. Although these protocols are more robust than the conventional topology-based multicast schemes, the header overhead increases significantly as the group size increases, which prevents the scaling of these protocols and constrains these protocols to be used only for small multicast groups. Additionally, there is a need to efficiently manage the membership of a potentially large group, obtain the positions of the members, and transmit packets to member nodes that may be located in a large network domain and in the presence of node movements. The existing small-groupbased geographic multicast protocols normally address only part of these problems.

In this paper, we propose a Robust and Scalable Geographic 
Multicast protocol (RSGM), which can scale to a large group size and network size and provide robust multicast packet transmissions in a dynamic mobile ad hoc network environment. The protocol is designed to be simple, thus it can operate more efficiently and reliably. We introduce several virtual architectures for more robust and scalable membership management and packet forwarding in the presence of high network dynamics due to unstable wireless channels and frequent node movements. Both the data packets and control messages will be transmitted along efficient tree-like paths, however, different from other tree-based protocols, there is no need to explicitly create and maintain a tree structure. A robust virtual-tree structure can be formed during packet forwarding with the guidance of node positions. Furthermore, RSGM makes use of position information to support reliable packet forwarding. The protocol is designed to be comprehensive and self-contained. Instead of addressing only a specific part of the problem, it introduces a zone-based scheme to efficiently handle the group membership management, and takes advantage of the membership management structure to efficiently track the locations of all the group members without resorting to any external location server. The zone structure is also formed virtually and the zone where a node is located can be calculated based on the node position and a reference origin. Different from conventional cluster structures, there is no need to involve a complicated scheme to create and maintain the zone. To avoid the need of network-wide periodic flooding of source information, we introduce Source Home to track the positions and addresses of all the sources in the network. In summary, our contributions in this work include:

- Proposing stateless distribution schemes that data packets and control messages can be sent along efficient virtualtree paths without the need of explicitly building and maintaining a tree-structure as in conventional tree-based multicast protocols. This greatly reduces the control overhead and increases the reliability and scalability of the protocol.

- Making use of the position information to design a scalable and reactive zone-based scheme for efficient membership management, which allows a node to join and leave a group quickly.

- Supporting efficient location search of multicast group members, by combining the location service with the membership management to avoid the need and overhead of using a separate location server.

- Introducing a Source Home to track the addresses and positions of the sources, to avoid network-wide periodic flooding of source information.

- Designing schemes to handle the empty-zone problems for both member zones and the Source Home, which are critical in designing a zone-based protocol.

- Making a detailed quantitative analysis of the per-node control overhead of the protocol, and performing extensive simulations to show the scalability and robustness of the protocol.

We organize the rest of this paper as follows. In Section II, we discuss some related work on MANET multicast proto- cols. We present a detailed design of the RSGM protocol in Section III. We quantitatively analyze the per-node control overhead of RSGM in Section IV, and present our simulations results in Section V to demonstrate the scalability and robustness of the protocol. We make further discussions on additional issues to be considered in Section VI, and conclude the paper in Section VII.

\section{RELATED WORK}

In this section, we first summarize the basic procedures assumed in conventional multicast protocols, and then discuss a few geographic multicast algorithms proposed in the literature.

As introduced in Section I, conventional topology-based multicast protocols include tree-based protocols (e.g., [13], [19], [24], [25]) and mesh-based protocols (e.g., [4], [12]). Tree-based protocols construct a tree structures for more efficient forwarding of packets to all the group members. Mesh-based protocols expand a multicast tree with additional paths that can be used to forward multicast data packets when some of the links break. A topology-based multicast protocol generally has the following three inherent components that make them difficult to scale:

- Group membership management. The group membership changes frequently as each node may join or leave a multicast group randomly, and the management becomes harder as the group size or network size increases.

- Creation and maintenance of a tree- or mesh-based multicast structure. The tree-based structures are difficult to maintain in the presence of the movement of nodes and the change of multicast group membership, while the mesh-based schemes achieve the robustness at the cost of extra network resource consumption.

- Multicast packet forwarding. The multicast packets are forwarded along the pre-built tree or mesh structures, which are vulnerable to breakage over the dynamic topology, especially in a large network with potentially longer paths.

Although efforts were made to develop more scalable topology-aware protocols [11], the topology-based multicast protocols are generally difficult to scale to a large network size, as the construction and maintenance of the conventional tree or mesh structure involve high control overhead over a dynamic network. The work in [27] attempts to improve the stateless multicast protocol [8], which allows it a better scalability. In contrast, RSGM uses a location-aware approach for more reliable membership management and packet transmissions. As the focus of our paper is to improve the scalability of location-based multicast, a comparison with topology-based protocols is out of the scope of this work. However, we note that at the similar mobility and system set-up, RSGM has a much higher packet delivery ratio than that of [27].

Besides the three components included in conventional topology-based multicast protocols, a geographic multicast protocol also requires a location service to obtain the positions of the members. The geographic multicast protocols presented in [16], [17] and [18] need to put the information of the entire tree or all the destinations into packet headers, which 
would create a big header overhead when the group size is large and constrain these protocols to be used only for small groups. In DSM [16], each node floods its location in the network. A source constructs a Steiner tree and encodes the multicast tree into each packet, and delivers the packet by using source routing. LGT [17] requires each group member to know the locations of all other members, and proposes two overlay multicast trees: a bandwidth-minimizing LGS tree and a delay-minimizing LGK tree. In PBM [18], a multicast source node finds a set of neighboring, next-hop nodes and assigns each packet destination to one next-hop node. The next-hop nodes, in turn, repeat the process. In GMP [5], which proposed for sensor networks, a node needs to perform a centralized calculation for more efficient tree construction. Therefore, it is more applicable for a smaller group in a static network.

The HRPM [6] and SPBM [20] are more related to our work, as the two share the essence as RSGM in improving the scalability of location-based multicast by using hierarchical group management. HRPM decomposes a large group into a hierarchy of recursively organized manageable-sized subgroups, and uses distributed geographic hashing to construct and maintain such a hierarchy. Although it is interesting to apply hashing to find the rendezvous point (RP) for the network to store and retrieve state information, the hashed location is obtained with the assumption of the network size, which is difficult for a dynamic network. Also, as the hashed location is virtual, it is possible that the nodes could not find the (consistent) RP. This can happen when a message (e.g., Join) reaches a node whose transmission range covers the virtual point, but the node is neither the one closest to the RP, nor aware of the node (which may be out of its transmission range) closest to the RP. The mobility of nodes will introduce additional challenge to the protocol, which may not only result in frequent RP handoff, but also increase the chance of RP search inconsistency and failure. Additionally, requiring a node to contact RP first for a Join will increase joining delay. It is also not clear how the membership change of a cell is known to the source during the transmission. In contrast, RSGM does not make any assumption of the network size in advance. Instead of using one RP as a core for group membership management, which may lead to a point of failure, RSGM introduces the Source Home to facilitate the quick finding of a resource, which is much more stable than a single point, and manages group membership more efficiently at the local range. Instead of simply using the overlay-based transmissions, RSGM assumes various aggregation techniques to forward packets along more efficient transmission paths. We did not directly compare our work with HRPM, as we do not know the hashing algorithm used and a different hashing scheme would lead to very different RP distribution and performance. However, we evaluated the performance of RSGM using a much larger network size and much lower node density, while geometric methods are known to work better in an environment with a higher node density as confirmed by our results.

SPBM, a Scalable Position-Based Multicast (SPBM) protocol, was proposed to improve the scalability of the protocol to group size. The network terrain is divided into a quad- tree with L levels. The top level is the whole network and the bottom level is constructed by basic squares. Each higher level is constructed by larger squares with each square covering four smaller squares at the next lower level. All the nodes in a basic square are within each other's transmission range. A node periodically broadcasts its membership and position in a basic square. At each level, every square needs to periodically flood its membership into its upper level square. Such periodic flooding is repeated for every two neighboring levels and the top level is the whole network region. Significant control overhead will be generated when the network size increases as a result of membership flooding. With this proactive and periodic membership updating scheme, the membership change of a node may need to go through L levels to make it known to the whole network, which leads to a long multicast group joining time. Instead, RSGM uses more efficient zone-based structure to allow nodes to quickly join and leave the group. Additionally, RSGM introduces Source Home to facilitate quick source discovery and avoid network-wide flooding of source information. As RSGM does not use any periodic network-wide flooding and uses stateless virtual-tree-based structures for control and data transmissions, RSGM can be scalable to both the group size and the network size.

\section{Robust and Scalable Geographic Multicast PROTOCOL}

In this section, we describe the RSGM protocol in details. RSGM supports a two-tier membership management and forwarding structure. At the lower tier, a zone structure is built based on position information and a leader is elected on demand when a zone has group members. A leader manages the group membership and collects the positions of the member nodes in its zone. At the upper tier, the leaders of the member zones report the zone membership to the sources directly along a virtual reverse-tree-based structure. If a leader is unaware of the position or addresses of the source, it could obtain the information from the Source Home. With the knowledge of the member zones, a source forwards data packets to the zones that have group members along the virtual tree rooted at the source. After the packets arrive at a member zone, the leader of the zone will further forward the packets to the local members in the zone along the virtual tree rooted at the leader.

Many issues need to be addressed to make the protocol fully functional and robust. The issues related to zone management include: the strategy for electing a zone leader on-demand and maintaining the zone leader during mobility, the handling of empty zone problem, the scheme for Source Home construction and maintenance, and the need to reduce packet loss during node moving across zones. The issues related to packet forwarding include: the scheme for virtual tree construction without the need of storing and tracking tree-state information, and the reliable transmissions of control and multicast data packets without resorting to an external location server.

For presentational convenience, we will first introduce the assumption made and the terminologies to be used in the rest of the paper. We assume every node is aware of its own position (e.g., through GPS or some in-door localization 


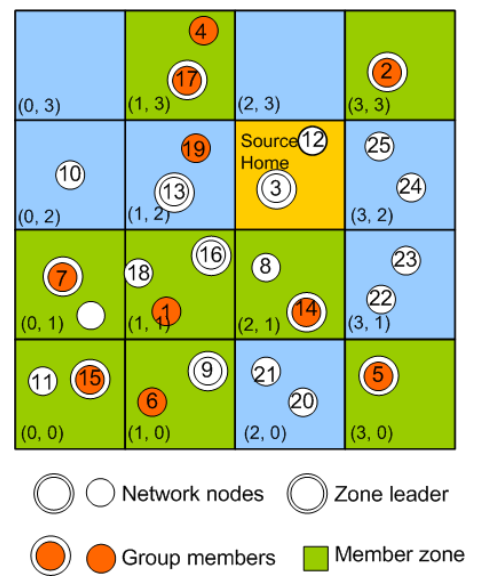

Fig. 1. A reference zone structure used in RSGM.

technique). The forwarding of data packets and most control messages is based on a geographic unicast routing protocol. In our performance study, we implemented GPSR [14] as an underlying unicast protocol to support the packet transmissions. The protocol, however, does not depend on a specific geographic unicast routing protocol.

Some of the notations to be used are:

pos: A mobile node's position coordinates (x, y).

zone: The network terrain is divided into square zones as shown in Fig. 1. We will study the impact of zone size on the performance of the protocol in Section V-B1.

mZone (non_mZone): Member (Non_member) zone, a zone with (without) group members in it.

$z L d r$ : Zone leader.

sHome: Source Home. A zone in the network is elected as Source Home to keep track of the addresses and locations of all the sources. To reduce the bottleneck problem and avoid routing inefficiency, the Source Home does not serve as the gateway for data forwarding between the source and group members.

groupID: The address of a multicast group.

mcastTable: Multicast table. A node records the multicast information in its multicast table, which contains a list of group entries, and the information on the Source Home including the identification and sequence number of the Source Home, which will be introduced later. Each group entry saves the information of a group: (groupID, source list, member list, mZone list). Source list is a list of source records, which is used by group members and zone leaders to keep the information of the sources. The member list is used by a zone leader to save the information of multicast group members within its local zones, and the source will record member zones in its zone list.

In this section, we will first introduce our zone construction and maintenance scheme in Section III-A, we will then present the group membership management scheme of RSGM in Section III-B. In Section III-C, we will describe how a session is initiated and an efficient source tracking strategy. Finally, in Section III-D, we will present our reliable packet forwarding scheme.

\section{A. Zone Construction and Maintenance}

In RSGM, the zone-structure is virtual and calculated based on a reference point. Therefore, the construction of zone structure does not depend on the shape of the network region, and it is very simple to locate and maintain a zone. To further reduce management overhead, a zone needs to elect a leader and be managed only when it has multicast group members.

1) Zone construction: Virtual zones are used as references for the nodes to find their zone positions in the network domain. The zone is set relative to a virtual origin located at $\left(x_{0}, y_{0}\right)$, which is set at the network initialization stage as one of the network parameters. The length of a side of the zone square is defined as zone size. Each zone is identified by a zone ID (zID). A node can calculate its $\operatorname{zID}(a, b)$ from its pos $(\mathrm{x}, \mathrm{y})$ as follows:

$$
\left\{\begin{array}{l}
a=\left[\frac{x-x_{0}}{z \text { zone_size }}\right], \\
b=\left[\frac{y-y_{0}}{z \text { zone_size }}\right] .
\end{array}\right.
$$

For simplicity, we assume all the zone IDs are positive. A zone ID will help locate a zone, and a packet destined to a zone will be forwarded towards its center. The center position $\left(x_{c}, y_{c}\right)$ of a zone with zID (a,b) can be calculated as:

$$
\left\{\begin{array}{l}
x_{\text {center }}=x_{0}+(a+0.5) \times \text { zone_size }, \\
y_{\text {center }}=y_{0}+(b+0.5) \times \text { zone_size. }
\end{array}\right.
$$

2) On-demand leader election: A leader will be elected in a zone only when the zone has group members in it to avoid unnecessary management overhead. When a multicast group member $\mathrm{M}$ just moves into a new zone, if the zone leader $(\mathrm{zLdr})$ is unknown, $\mathrm{M}$ queries the neighbor node in the zone for the leader. When failing to get the leader information, $\mathrm{M}$ will announce itself as a leader by flooding a LEADER message into the zone. In the case that two leaders exist in a zone, e.g., due to the slight time difference of leader queries and announcements, the one with the larger ID will win and be selected as the leader. A zone leader floods a LEADER in its zone every time interval Intval refresh $_{\text {to }}$ announce its leadership until the zone no longer has any members. If no LEADER message is received within the interval $2 \times$ Intval $_{\text {refresh }}$, a member node will wait for a random period and then announce itself as the zone leader when no other node announces the leadership.

\section{B. Group Membership Management}

The group membership is managed at two tiers. RSGM takes advantage of the virtual-zone-based structure to efficiently track the group membership and member positions. In the following description, except when explicitly indicated, we use $\mathrm{G}, \mathrm{S}$ and $\mathrm{M}$ respectively to represent a multicast group, a source of $\mathrm{G}$ and a member of $\mathrm{G}$.

1) Local group membership management: The group membership is first aggregated in the local zone and managed by the zone leader. When joining or leaving a group, a member $\mathrm{M}$ sends a message REFRESH (groupIDs, pos $_{M}$ ) immediately to its zone leader to notify its membership change, where pos $M$ is its position and groupIDs are the addresses of the groups in 
which $\mathrm{M}$ is a member. $\mathrm{M}$ also needs to unicast a REFRESH message to its zone leader every time interval Intval refresh $_{\text {r }}$ to update its position and membership information. A member record will be removed by the leader if not refreshed within $2 \times$ Intval $_{\text {refresh }}$.

When M moves to a new zone, its next periodic REFRESH will be sent to the zone leader in the new zone. It will announce itself as the leader if the new zone does not have one. The moving node will still receive the multicast data packets from the old zone before its information is timed out at the leader of the old zone, which reduces the packet loss during the moving. For a leader node, if its distance to the zone border is shorter than a distance threshold and the zone is still a member zone, it will handover its leadership by unicasting a LEADER message (carrying all the current group information) to the neighbor node in its zone which is closest to the zone center. The LEADER message will continue being forwarded towards the zone center until reaching a node which has no neighbor closer to the zone center than itself, and the node will take over the leadership and flood a LEADER within the zone.

2) Membership management at the network level: After the membership information is aggregated in the local zone, a source only needs to track the IDs of the member zones that have group members. The leaders of the member zones are responsible for the sending of the zone membership information to the source.

a) Zone membership reporting by zone leaders

When a zone changes from a member zone to a non member zone of $\mathrm{G}$ or vice versa, the zone leader sends a REPORT message immediately to $S$ to notify the change. The leader can obtain the address and position of $\mathrm{S}$ using methods described in Section III-C. A zone leader needs to send REPORT every time interval Intval zone $_{\text {ene }}$ to $\mathrm{S}$ to refresh its zone membership information. In the case that $\mathrm{S}$ is the source of more than one multicast group, instead of sending a REPORT to $S$ for each group, the leader sends one REPORT carrying all corresponding group IDs. S will remove a member-zone record if not refreshed within $2 \times$ Intval $_{z o n e}$.

b) Empty zone handling

A zone may become empty when all the nodes move away. The probability that a zone is empty is approximately $P=e^{-\rho r^{2}}$ when the node density is $\rho$ and the zone size is $r$. Let's calculate the probability of zone being empty for two typical node densities and zone sizes: 1) When $\rho=60$ nodes $\left./ \mathrm{km}^{2}, r=100 \mathrm{~m}, P=0.55 ; 2\right)$ When $\rho=20$ nodes $/ \mathrm{km}^{2}, r=400 \mathrm{~m}, P=0.04$. We can see that in either case, the probability of a zone being empty is not negligible. Therefore, it is critical to address the empty-zone problem.

When a member zone of $\mathrm{G}$ is becoming empty, the moving out zone leader will notify $\mathrm{S}$ immediately to stop sending packets to the empty zone. If the moving out leader fails to notify S (e.g., the leader suddenly dies), the packet forwarded to the empty zone will finally be dropped without being delivered. The node which drops the packet will notify $S$ to delete the zone from its zone list. A false deletion will be corrected when $\mathrm{S}$ receives the periodic membership reporting again from the corresponding zone.

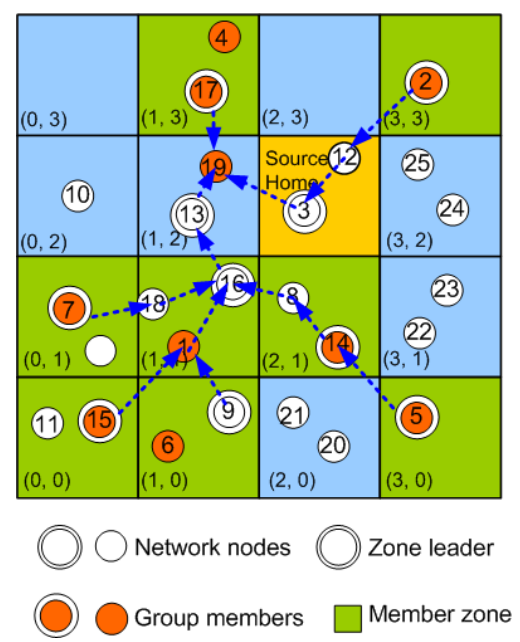

Fig. 2. The aggregation of REPORT messages and the virtual-reverse-tree formulation.

\section{c) Message aggregation}

As compared to local messages, control messages sent at the network tier would generally traverse a longer path. To minimize control overhead, we consider a virtual reversetree-based aggregation scheme (Fig. 2), with which all the control messages sent towards the same destination (e.g., the source S) will be aggregated to further reduce control overhead. Different from other tree-based multicast protocols, no explicit tree-structure needs to be maintained, which avoids the overhead and improves the robustness. Specifically, the periodic REPORT messages can be aggregated and forwarded along the reverse tree. To facilitate the message aggregation, $\mathrm{S}$ schedules the periodic REPORT sending for the member zones. $\mathrm{S}$ inserts the next periodic reporting time $t$ into the data packets sent out. The leader of a member zone schedules its next periodic REPORT to $\mathrm{S}$ at time $t+\Delta t$, where $\Delta t$ is inversely proportional to its distance to $\mathrm{S}$. The zone leaders will form an upstream and downstream relationship according to their distances to S. Generally the leaders farther away from $\mathrm{S}$ have a shorter $\triangle t$ and will send the REPORTs earlier than the upstream zone leaders, while strict timing is not needed. When a REPORT message reaches a member zone, it is forwarded to the leader first. When an upstream zone leader receives REPORTs from downstream zone leaders, if it has not sent out its REPORT, it will aggregate these REPORTs with its own REPORT, and send out the REPORT at its scheduled time. As a result, the forwarding of the REPORT messages follows a tree structure as shown in Fig. 2. The REFRESH messages sent by member nodes to the zone leader can be similarly aggregated and sent through the virtual reverse tree.

\section{Session Initialization and Source Tracking}

In order to join and leave a multicast group, the nodes in the network need to have the source information. As a source can move in a MANET, it is critical to quickly find the source when needed and efficiently track the location of the source node. RSGM incorporates mechanisms for session creation and efficient source discovery. 
1) Session initiation: A multicast session (G) is initiated and terminated by a source (S). To start a multicast session, $\mathrm{S}$ floods an ANNOUNCE (S, pos ${ }_{S}$, groupIDs) message into the network (for reliability, promiscuous broadcasting is used in the flooding), where groupIDs are IDs of the groups ( $\mathrm{G}$ is one of them) for which $\mathrm{S}$ is the source. Upon receiving this message, a node $(\mathrm{N})$ interested in being the group member of $\mathrm{G}$ starts the joining process by unicasting to its zone leader a REFRESH message carrying the information of $\mathrm{S}$. After a session begins, $S$ can piggyback its position $\left(\operatorname{pos}_{S}\right.$ ) to the multicast packets sent out to refresh its position at the receivers. When a member $M$ moves to a new zone, the new leader can obtain the address and position of $\mathrm{S}$ from $\mathrm{M}$. To terminate $\mathrm{G}, \mathrm{S}$ floods an ANNOUNCE message with $\mathrm{G}$ removed from its group ID list.

2) Source tracking: A source may move during the session time. The forwarders and receivers of the multicast packets can obtain the position of the source that is piggy-backed with the packets, while other nodes including the ones that newly join the network must resort to some explicit source location or update mechanism to get the position. The conventional scheme for resource information update is through periodic network-wide flooding of source information [12]. Straightforward ways to look for a source include flooding query messages, and performing an expanding ring search. However, these methods will incur excessive control overhead and search delay.

To facilitate the source location and avoid network-wide periodic flooding of source information, we introduce a Source Home, a zone in which all the nodes will keep track of the multicast sources in the network. To avoid being a bottleneck, increase survivability, and improve transmission efficiency, the Source Home will not serve as the gateway for data traffic to the source. The issues related to the management of Source Home are: 1) creation and maintenance of the Source Home with reliability, uniqueness and consistency; 2) efficient information update to the Source Home.

When a new source is started and does not know the zone ID of the Source Home, it will perform an expanding ring search within a smaller range. Compared to a single node, the location of the Source Home will be relatively stable and can be cached by network nodes. If no Source Home is located, it will announce its current zone as Source Home by flooding an ANNOUNCE message into the network with the sequence number of the Source Home set to zero. All the network nodes will record the zone ID and sequence number of the Source Home. Later multicast sources will share the elected Source Home and all the nodes in the Source Home will maintain the addresses and IDs of the sources. Whenever a source moves to a new zone, it unicasts a REGISTER $\left(z I D_{\text {new }}\right)$ message to the Source Home. When the message reaches the Source Home, the first node receiving it floods the message into the Source Home so that all the nodes learn which zone the source is currently located in. To learn the source information which is currently maintained by the Source Home, a node just moving into the Source Home will query its neighbors in the zone. During the zone membership reporting (Section III-B2), a zone leader will send a REPORT message to the Source Home if it does not know the source address or the address it maintains is outdated. The first node in the Source Home that receives the REPORT and has a record of $\mathrm{S}$ will forward the message towards the zone where $\mathrm{S}$ is located. When the REPORT message arrives at the zone of $S$, the message will be first forwarded to the leader. As $S$ is a member of $G$ and needs to send REFRESH periodically to its leader, the leader has the position of $\mathrm{S}$ and will forward the packet to $\mathrm{S}$.

If the Source Home is becoming empty, when a leaving node finds it has no neighbors in the zone, it will announce its entering zone as the new Source Home to the network, and flood into the new Source Home its source list which contains the information of the sources it currently maintains. The sequence number of the Source Home is increased by one every time the Source Home changes. Some nodes may have no information on the Source Home or hold an old zone ID due to their failing to receive the announcement of the updated Source Home. To handle the first case, a node can learn the source-home information by an expanded ring search. For the second case, the sequence number can help a node to identify the newest Source Home. A message sent to the Source Home (e.g., REGISTER message) will carry the sequence number. A forwarding node will update its recorded source-home information if the sequence number carried by the incoming message is larger than that it has; otherwise, it forwards this message to its recorded Source Home, and sends back the source-home update information to the sending node if the sequence number carried by the incoming message is smaller than that it has. The sequence number will also help elect a Source Home when multiple ones exist. This can occur if a source does not know or cannot reach the Source Home, and announces its own zone as Source Home again. The Source Home with the larger sequence number wins or the one with the larger ID wins when the two zones have the same sequence number, and the holder of the invalid source-home information will be notified as described above. As an example, in the case of network partitioning, a source may announce its current zone as new Source Home since it cannot reach the Source Home. When the network is connected again, multiple Source Homes exist, and one Source Home will be elected through our Source Home maintenance scheme. This source-home merging process will also help merge the partitioned group resulted from network partition.

With a Source Home, there is no need to flood the source information to the network periodically or search for Source throughout the network, which greatly reduces the management overhead and multicast group joining delay.

\section{Multicast Packet Delivery}

A source needs to send the multicast packets reliably to the group members. With the membership management, the member zones are recorded by source $S$, while the local group members and their positions are recorded by the zone leaders. Multicast packets will be sent along a virtual distribution tree from the source to the member zones, and then along a virtual distribution tree from the zone leader to the group members. A virtual distribution tree is formulated during transmission time and guided by the destination positions. 
The multicast packets are first delivered by $\mathrm{S}$ to member zones towards their zone centers. S sends a multicast packet to all the member zones, and to the member nodes in its own zone through the zone leader. For each destination, it decides the next hop by using the geographic forwarding strategy described in Section I. After all the next hops are decided, $\mathrm{S}$ unicasts to each next hop node a copy of the packet which carries the list of destinations that must be reached through this hop. Only one copy needs to be sent when packets for different destinations share the same next hop node. Thus the packets are forwarded along a tree-like path without the need of building and maintaining the tree in advance. For robust transmissions, geographic unicast is used in packet forwarding. The packets can also be sent through broadcast to further reduce forwarding bandwidth, at the cost of reliability.

When an intermediate node receives the packet, if its zone ID is not in the destination list, it will take a similar action to that of $\mathrm{S}$ to continue forwarding the packet. If its zone is in the list, it will replace its zone ID in the destination list with the local members if it is a zone leader, or replace the ID with the position and address of the zone leader otherwise. The intermediate node will find the next hop node to each destination and aggregate the sending of packets that share the same next hop node as source $\mathrm{S}$ does.

\section{Cost Analysis}

In this section, we quantitatively analyze the per node cost of the protocol, which is defined as the average number of control messages transmitted by each node per second. We will analyze the basic two-tier scheme, and for simplicity, in most cases, we will not consider the message aggregations, thus the analysis result is an upper bound of the cost.

TABLE I

NOTATIONS USED IN THE COST ANALYSIS

\begin{tabular}{|c|c|}
\hline$N$ & total number of mobile nodes within the network \\
\hline$r$ & zone size \\
\hline$r_{t}$ & transmission range \\
\hline$R$ & $\begin{array}{l}\text { network size, assuming a square network terrain with a side } \\
\text { length } R\end{array}$ \\
\hline$v$ & average moving speed of the mobile nodes \\
\hline$G$ & total number of multicast groups \\
\hline$S$ & total number of sources \\
\hline$M_{n}$ & total number of member nodes \\
\hline
\end{tabular}

The notations to be used in this section are listed in Table I. With a two-tier system structure, the total cost includes the cost for upper tier management and the cost for lower tier management. Before obtaining the cost of the overall protocol, we first introduce a few lemmas, and calculate the per-node control overhead for each tier.

Lemma 1: Assume that a node keeps the same moving direction in a zone. Then the average moving distance of the mobile nodes in a zone is $\frac{\pi r}{4}$.

Proof: The moving distance $d$ of a node in a zone is the length of its moving trail in the zone square. For example, in Fig. 3, line $a$ is such a moving trail. Suppose the angle formed by the moving trail and the bottom side of the zone

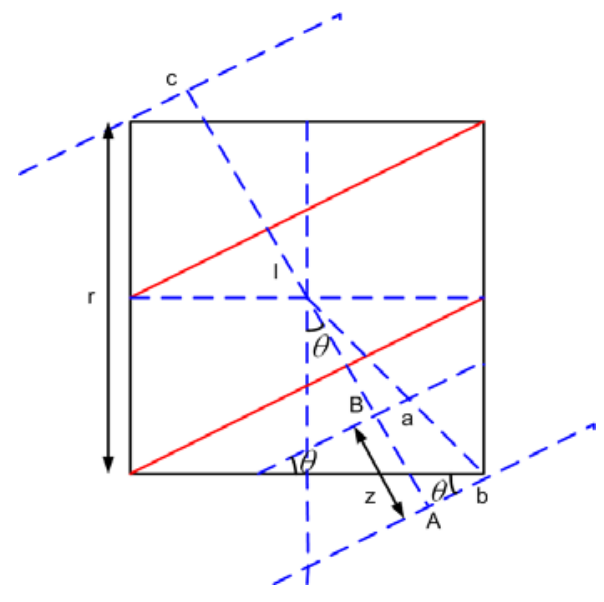

Fig. 3. The moving distance of a mobile node in a zone.

square is $\theta$. Due to the symmetry of the square, we only need to consider the case when $\theta \in\left[0, \frac{\pi}{4}\right]$. As illustrated in Fig. 3, all the possible moving trails with angle $\theta$ are located between two parallel lines $b$ and $c$, where $b$ and $c$ are tangent to the zone with angle $\theta$. Line $l$ is perpendicular to $b$ and $c$ and intersects $b$ at point $A$. $a$ intersects $l$ at $B$. If the distance between $A$ and $B$ is $z$, the length of a moving trail is decided by its angle $\theta$ and distance $z$. Therefore, we can calculate the average distance of a node moving in a zone as

$$
\begin{aligned}
d & =\frac{\int_{0}^{\frac{\pi}{4}}\left(2 \int_{0}^{r \sin \theta} \frac{z}{\sin \theta \cos \theta} d z+\int_{r \sin \theta}^{\frac{r}{2}(\cos \theta-\sin \theta)} \frac{r}{\cos \theta} d z\right) d \theta}{\int_{0}^{\frac{\pi}{4}} \int_{0}^{\frac{r}{2}(\cos \theta+\sin \theta)} d z d \theta} \\
& =\frac{\pi r}{4} .
\end{aligned}
$$

Lemma 2: The per node cost of RSGM due to lower tier management is $\mathrm{O}(1)$ with respect to the network size and group size.

Proof: The overhead of the lower tier management comes from the REFRESH messages sent by non-leader members and the LEADER messages from leaders of member zones.

A member node unicasts a REFRESH to its zone leader

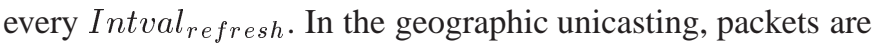
normally greedily forwarded and the perimeter forwarding is only in the recovery mode. For simplicity, we only consider the greedy forwarding. According to [23], the average number of hops of the greedy forwarding path between the source and the destination is $\frac{\bar{d}}{\bar{z}}$, where $\bar{d}$ is the average distance between the source and destination and $\bar{z}$ is the average forwarding progress made towards the destination in the course of one transmission, which depends on $r_{t}$ and the average number of nodes within $r_{t}$. The average distance between two nodes in the same zone is $\frac{\pi r}{8}$ by Lemma 1 . Therefore,

Cost $_{\text {REFRESH }}=\frac{1}{N} \frac{M_{n} \pi r}{8 \bar{z} \times \text { Intval }_{\text {refresh }}}=O\left(\frac{M_{n}}{N}\right)=O(1)$

A leader of the member zone floods a LEADER message in the local zone every Intval refresh $_{\text {f }}$ and unicasts a LEADER to another node to handover the leadership when leaving the zone. Because there is an average of $\frac{N r^{2}}{R^{2}}$ nodes in a zone, the 
average number of transmissions due to local zone flooding is $\left(\frac{N r^{2}}{R^{2}}-1\right)$. By Lemma 1 , the average frequency of a node moving between different zones is $\frac{4 v}{\pi r}$. Therefore,

$$
\begin{aligned}
\operatorname{Cost}_{L E A D E R} & =\frac{1}{N}\left(\frac{M_{z}}{\text { Intval }_{\text {refresh }}}\left(\frac{N r^{2}}{R^{2}}-1\right)+M_{z} \frac{\pi r}{8 \bar{z}} \frac{4 v}{\pi r}\right) \\
& =O\left(\frac{1}{N}\left(N+M_{z}\right)\right), \because M_{z} \leq \frac{R^{2}}{r^{2}} \\
& =O(1) .
\end{aligned}
$$

From Eq. (4) and Eq. (5), the per node cost of the lower tier management with respect to the network size and group size is:

$$
\text { Cost }_{\text {lower }}=\text { Cost }_{\text {REFRESH }}+\text { Cost }_{\text {LEADER }}=O(1) .
$$

Lemma 3: The per node cost of RSGM for the upper tier management is $\mathrm{O}(1)$ with respect to the network size and group size.

Proof: The overhead of the upper tier management is the sum of the overhead of REPORT messages, REGISTER messages and Source Home management messages.

The leader of a member zone unicasts a REPORT to the source of each multicast group every Intval $_{\text {zone }}$. We analyze the overhead in the worst case when all the zones are member zones and there is no empty zone. In this case, when message aggregation is used, a REPORT sent by a zone leader will stop at an upstream zone leader. When $r \geq r_{t}$, the upstream zone is one of its neighboring zones, and the distance between the two leaders is shorter than $2 \sqrt{2} r$. In the case of $r<r_{t}$, the upstream zone may not be its neighboring zone, and the distance between the two leaders will be shorter than $2 \sqrt{2} r+$ $r_{t}$. Hence,

$\operatorname{Cost}_{R E P O R T}<\frac{1}{N} \frac{M_{z} G\left(2 \sqrt{2} r+r_{t}\right)}{\bar{z} \times \text { Intval }_{\text {zone }}}=O\left(\frac{M_{z}}{N}\right)=O(1)$.

Whenever a source moves into a new zone, it unicasts a REGISTER to the Source Home, and then the REGISTER is flooded in the Source Home. Hence,

Cost $_{\text {REGISTER }}=\frac{1}{N}\left(\frac{\pi R}{8 \bar{z}}+\frac{N r^{2}}{R^{2}}-1\right) S \frac{4 v}{\pi r}=O\left(\frac{R}{N}\right)$,

and since $N=d R^{2}$, where $\mathrm{d}$ is the node density,

$$
\text { Cost }_{R E G I S T E R}=O\left(\frac{1}{R}\right)=O(1), \text { when } R \geq 1 .
$$

Whenever the Source Home becomes empty, the last leaving node will announce its current zone as the Source Home by flooding into the network an ANNOUNCE message, and flood into the new Source Home a SOURCE message. The cost for this part is:

$$
\text { Cost }_{1} \leq \frac{1}{N}\left(N+\frac{N r^{2}}{R^{2}}-2\right) \frac{4 v}{\pi r}=O(1) .
$$

When a node moves into the Source Home, it broadcasts a message to query the source list, and one of its neighbors sends back a message to reply. Since there is an average of $\frac{N r^{2}}{R^{2}}$ nodes in a zone and the average time a node stays in a zone is $\frac{\pi r}{4 v}$, we consider the worst case that during a time interval of $\frac{\pi r}{4 v}$, there are $\frac{N r^{2}}{R^{2}}$ nodes moving in the Source Home. Hence,

$$
\text { Cost }_{2}<\frac{1}{N} \frac{2 N r^{2}}{R^{2}} \frac{4 v}{\pi r}=O(1) .
$$

Therefore, the cost for Source Home management is:

$$
\text { Cost }_{\text {some }}=\text { Cost }_{1}+\text { Cost }_{2} \leq O(1) .
$$

From Eq. (7), Eq. (8) and Eq. (9), the per node cost of the upper tier management with respect to the network size and group size is:

$$
\begin{aligned}
\text { Cost }_{\text {upper }} & =\text { Cost }_{\text {REPORT }}+\text { Cost }_{\text {REGISTER }}+\text { Cost }_{\text {sHome }} \\
& =O(1) .
\end{aligned}
$$

Theorem 1: The RSGM control overhead as the average number of control message transmissions per node every second has a complexity of $\mathrm{O}(1)$ with respect to the network size and group size.

Proof: The overhead of the protocol is caused by the lower tier management, the upper tier management and the periodic beaconing in the underlying geographic unicast routing protocol. The cost of unicast is:

$$
\text { Cost }_{\text {unicast }}=\frac{1}{\text { Intval }_{\text {beacon }}}=O(1) \text {. }
$$

By Lemma 2, Lemma 3 and Eq. (11), the cost of the protocol, i.e., the transmissions of control messages per node every second with respect to the network size and group size is:

Cost $_{\text {protocol }}=$ Cost $_{\text {lower }}+$ Cost $_{\text {upper }}+$ Cost $_{\text {unicast }}=O(1)$.

The analysis result shows that when the network size and group size increase, the control overhead placed on each node by the protocol will remain relatively constant. Next, we will demonstrate the scalability of the protocol by simulation studies.

\section{Performance Evaluation}

In this section, we study the performance of RSGM by simulations. We are mainly interested in the protocol's scalability and robustness in a dynamic environment.

\section{A. Simulation Overview}

We implemented RSGM within the Global Mobile Simulation (GloMoSim) [22] library. We implemented the geographic unicast protocol GPSR described in [14]. In GPSR, a source can obtain the destination position through some type of location service [10] [9]. An intermediate node makes its forwarding decisions based on the destination position inserted in the packet header by the source and the positions of its one-hop neighbors learned from the periodic beaconing of the neighbors. The protocol consists of two transmission modes. In the greedy mode, a forwarding node forwards the packet 
(a)

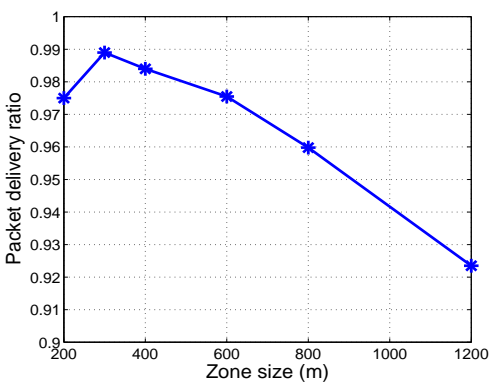

(c)

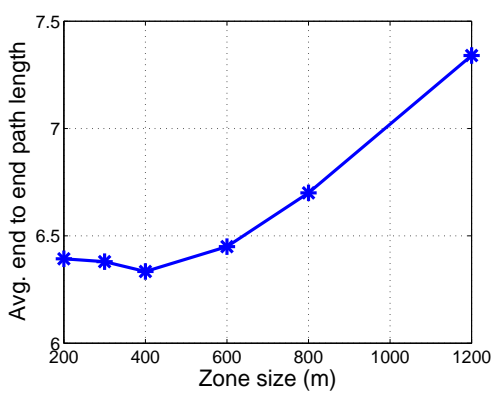

(b)

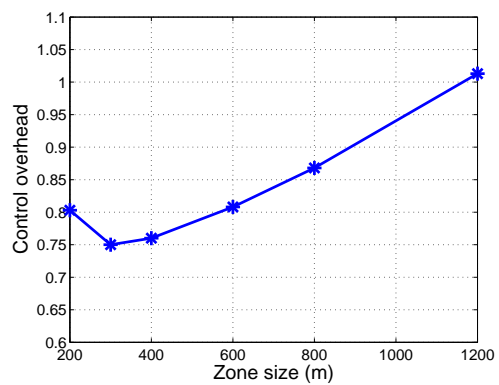

(d)

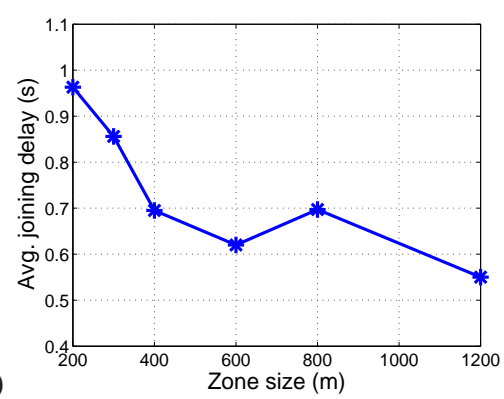

Fig. 4. RSGM performance vs. zone size (1 group, 1 source, 100 group members): (a) packet delivery ratio; (b) normalized control overhead; (c) average path length; (d) average joining delay.

to the neighbor that is closest to the destination and. When no such a neighbor exists, the node enters recover mode and assumes perimeter forwarding [14] to recover from the local void. In this case, a packet traverses the face of the planarized local topology subgraph by applying the right-hand rule until the greedy forwarding can be resumed. The implementation includes a proactive beaconing mechanism with promiscuous use of the network interface as in [14], and the beaconing interval was set as 4 seconds. We set RSGM's Intval refresh $_{\text {r }}$ as 4 seconds and Intval $_{z o n e}$ as 6 seconds. Except in the study of the impact of zone size, the zone size was set as 400 meters.

For performance reference, we choose to compare with the classic mesh-based, on-demand non-geographic multicast protocol ODMRP [12] as it is widely used and considered to be robust over a dynamic network, and geographic multicast protocol SPBM [20][21] which is designed to improve the scalability of position-based multicast. The SPBM is a quadtree-based protocol as introduced in Section II. ODMRP is a mesh-based on-demand non-geographic multicast protocol, and takes a soft-state approach to maintain multicast group members. A multicast source broadcasts a Join-Query messages to the entire network periodically. An intermediate node stores the source ID and the sequence number, and updates its routing table with the node ID (i.e. backward learning) from which the message was received for the reverse path back to the source. A receiver creates and broadcasts a Join Reply to its neighbors, with the next hop node ID field filled by extracting information from its routing table. The neighboring node whose ID matches that in the message broadcasts its own Join Table built upon matched entries. This whole process constructs (or updates) the routes from sources to receivers and builds a mesh of nodes, the forwarding group.

The simulations for ODMRP are based on the codes carried with the simulator, with parameters set as those in [15].
We fixed several bugs in the GloMoSim codes which would prevent a forwarding group node from sending JOIN TABLES. The improvement doubles the delivery ratio and reduces the control overhead of ODMRP. Additionally, we implemented SPBM in GloMoSim according to the protocol descriptions in [20][21] and the ns 2 codes provided by the authors, with the parameters set as those in [21]. The basic square size was set to $150 \mathrm{~m}$ so that the nodes in a basic square are within each others transmission range. The number of levels of the quadtree changes accordingly with the square size and the larger network size we used.

The simulations were run with 400 nodes randomly distributed in the area of $2400 \mathrm{~m} \times 2400 \mathrm{~m}$. The nodes moved following the modified random waypoint mobility model [7]. The minimum moving speed was set as 1 meter per second and the default maximum speed was set as 20 meters per second except when studying the effect of mobility by varying the moving speed. We set the MAC protocol and radio parameters according to the Lucent WaveLAN ${ }^{T M}$ card, which operates at a data rate $11 \mathrm{Mbps}$ and radio frequency $2.4 \mathrm{GHz}$ with a nominal transmission range of 250 meters. IEEE $802.11 \mathrm{~b}$ was used as the MAC layer protocol. Each simulation lasted 500 simulation seconds. Each source sends CBR data packets at $8 \mathrm{Kbps}$ with packet length 512 bytes. The CBR flows start at around 30 seconds so that the group membership management has time to initialize and stop at 480 seconds. The default group size was 100 members with one source per group. By default, one multicast group was simulated except when evaluating the performance of different numbers of groups in the network. A simulation result was gained by averaging over six runs with different seeds.

We are mainly interested in the protocol's scalability, robustness and efficiency under the dynamic environment. The following metrics were studied: 
(a)

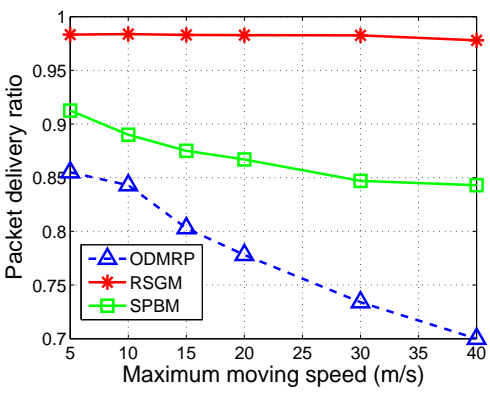

(c)

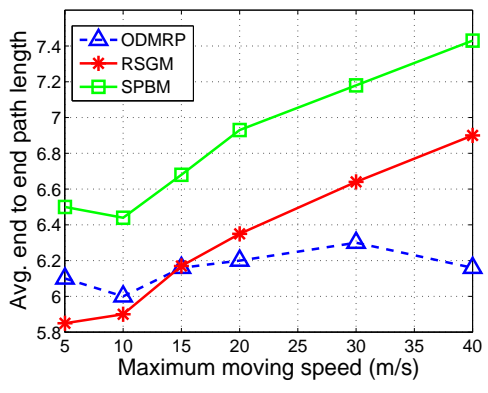

(b)

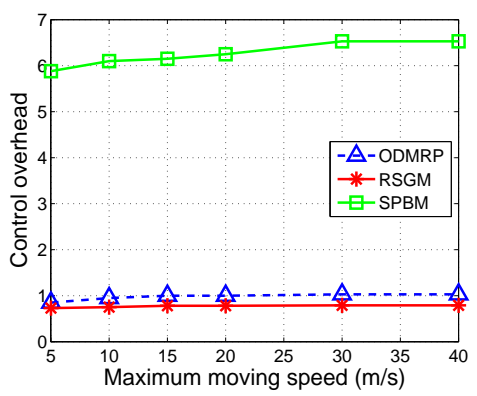

(d)

Fig. 5. Performance vs. maximum moving speed (1 group, 1 source, 100 group members): (a) packet delivery ratio; (b) normalized control overhead; (c) average path length; (d) average joining delay.

1) Packet delivery ratio: The ratio of the number of packets received and the number of packets expected to be received. For the multicast packet delivery, the ratio is equal to the total number of received packets over the multiplication of the group size and the number of originated packets.

2) Normalized control overhead: The total number of control message transmissions divided by the total number of received data packets. The control messages include the control messages of RSGM and the proactive beacons in the underlying geographic unicast routing protocol. Each forwarding of the control messages was counted as one transmission.

3) Average path length: The average number of hops traversed by each delivered data packet.

4) Joining delay: The average time interval between a member joining a group and its first receiving of the data packet from that group. To obtain the joining delay, the simulations were rerun with the same settings except that all the members joined groups after the sources began sending data packets.

\section{B. Simulation Results}

The performance of the protocol may be impacted by many factors. We first study the impact of zone size on the performance of RSGM, and then compare the performance of ODMRP, SPBM and RSGM with the variation of moving speed and node density. Finally, we study the scalability of the three protocols with the change of group size, the number of groups in the network and network size.

1) Impact of zone size: Although the basic zone size in SPBM is restricted so that all the nodes in a zone are within transmission range, the nodes in a zone of RSGM can be multiple hops away. We first study the impact of zone size on the performance of RSGM. The zone size not only impacts the number of group members in a zone but also impacts the number of member zones in the network. As shown in Fig. 4, a too small or too large zone size will both lead to the performance degradation. Hence, a medium zone size (e.g., $400 \mathrm{~m})$ is preferred for RSGM.

With a smaller zone size, the number of member zones will increase, leading to more REPORT messages at the hightier. The zone leaders will also move out of the zones more frequently and generate more LEADER messages to handover the leadership. The movement of member nodes across zones would also possibly lead to zone membership changes. In Fig. 4 (b), the control overhead first drops with the increase of zone size, and then rises quickly as the zone size increases beyond a certain value. A larger zone size will result in a higher control overhead in the local zone due to the periodic local flooding of the LEADER messages in the member zones. The higher control overhead will cause more collisions and hence result in more packet droppings, and the delivery ratio therefore decreases with a too small or too large zone size. However, the delivery ratio is higher than $92 \%$ under all zone sizes as shown in Fig. 4 (a).

With a larger zone size, a zone has more stable membership, thus the joining processes of more group members will end in the local zone. Therefore, the average joining delay reduces with the increase of zone size as demonstrated in Fig. 4 (d). However, a reverse trend is seen in Fig. 4 (c). The delivery path is shorter with a smaller zone size. In RSGM, a packet is first forwarded towards the center positions of member zones, and when it reaches a member zone, the packet will be forwarded to the zone leader first and then to the local members. With a larger zone size, a packet needs to traverse a longer distance in a zone to reach the zone leader, resulting in a longer average end-to-end path. 
(a)

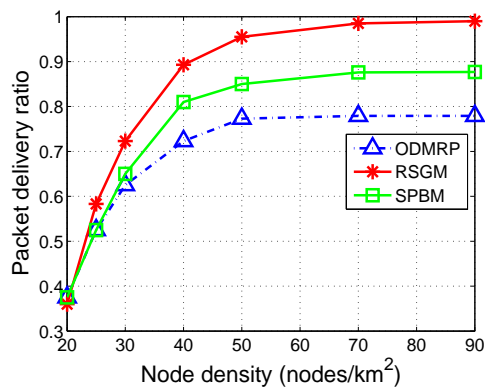

(c)

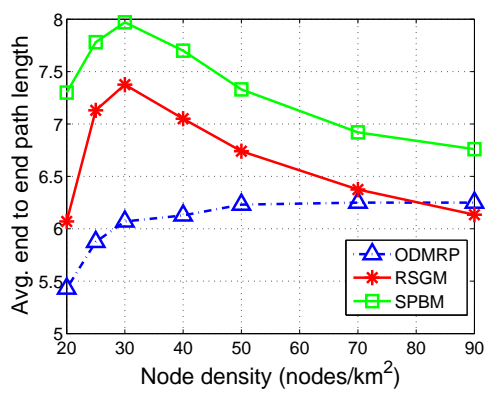

(b)

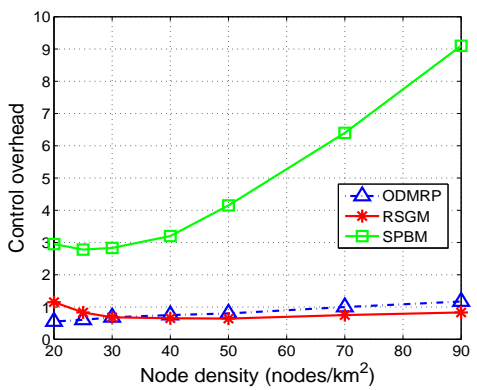

(d)

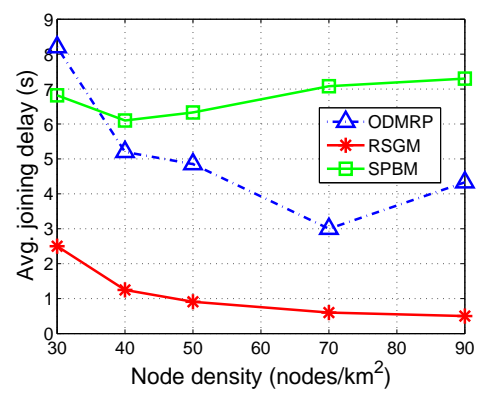

Fig. 6. Performance vs. node density (1 group, 1 source, 100 group members): (a) packet delivery ratio; (b) normalized control overhead; (c) average path length; (d) average joining delay.

(a)

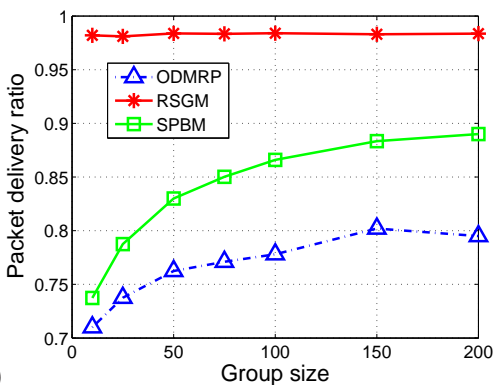

(c)

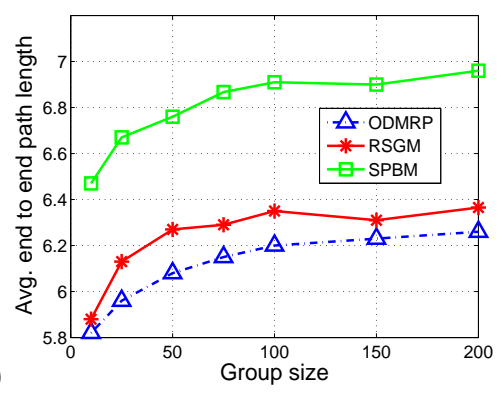

(b)

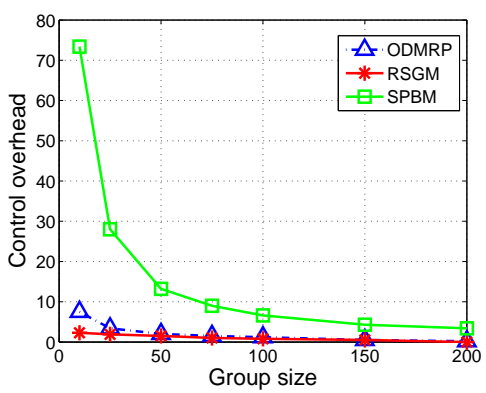

(d)

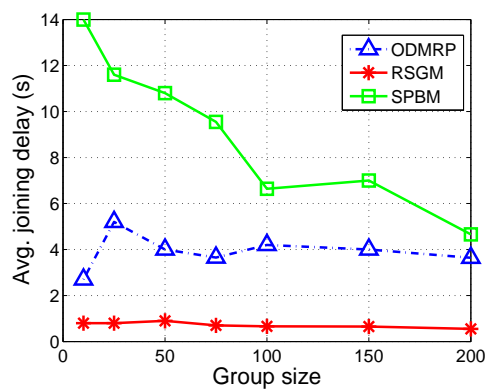

Fig. 7. Performance vs. group size (1 group, 1 source): (a) packet delivery ratio; (b) normalized control overhead; (c) average path length; (d) average joining delay.

2) Impact of mobility: It is critical and challenging for a multicast routing protocol to maintain a good performance in the presence of node mobility in an ad hoc network. We evaluate the protocol performance by varying maximum moving speed from $5 \mathrm{~m} / \mathrm{s}$ to $40 \mathrm{~m} / \mathrm{s}$.

From Fig. 5, in almost all the mobility cases, RSGM performs much better than ODMRP and SPBM. In all the mobility cases, the geographic multicast protocols RSGM and SPBM have higher delivery ratios. This is as expected, since geographic forwarding is more robust to the network topology change and both protocols use geographic unicast in their data packet transmissions to enhance reliability. RSGM keeps a stable and over $98 \%$ delivery ratio under all the mobility cases. The delivery ratios of ODMRP and SPBM decrease as mobility increases, and the delivery ratio of ODMRP drops much faster. Although the mesh structure used in ODMRP is more robust than the general tree structure, the mesh structure built through the back learning scheme is easier to become invalid as nodes move. In SPBM, when the mobility increases, its periodic multi-level membership update mechanism cannot catch the quick membership changes of the lower level squares in time, while its higher control overhead results in much more 
transmission collisions. Both factors lead to the reduction of its delivery ratio.

In Fig. 5(b), SPBM is seen to have a significantly higher overhead than the other two protocols due to its use of periodic local and network-wide flooding in its membership management. The control overhead of RSGM is seen to be the lowest. The membership management of RSGM is based on the efficient virtual-zone-based structure and supported with the inverted-virtual-tree for membership reporting without involving periodic network-wide flooding, which greatly improves the efficiency and scalability of the protocol. The control overhead of all the protocols increases as mobility increases. In RSGM, when the moving speed increases, there are more frequent leader changes and zone crossings, which triggers more handover processes. The increase of the normalized control overhead for all the protocols is also due to the reduced number of packets received in a highly dynamic environment.

The average path lengths of all the protocols increase in (Fig. 5 (c)), which indicates the delivery path will become nonoptimal sooner in a higher mobility environment. The mobility has more impact on the path lengths of the two geographic multicast protocols RSGM and SPBM. One reason is that the underlying geographic forwarding relies on periodic beaconing to refresh the positions of the neighbors, which cannot catch up with the changes of the neighbors' positions at a high moving speed, resulting in non-optimal forwarding decisions and longer routing paths as analyzed in work [26]. Another reason is that ODMRP has a shorter average end-to-end path under a higher mobility, as its pre-built paths (i.e., the mesh structure) especially those longer paths are more likely broken so packets with longer paths fail to reach their destinations. Between the two geographic multicast protocols, RSGM has a shorter path length. In RSGM, the packet forwarding from the source to a member zone follows the shortest path and a detour is only introduced in the destination zone by forwarding packets first to the zone leader and then to group members. In SPBM, the multicast packet forwarding follows its quad-tree structure and detours occur at multiple tree levels.

In RSGM, when a node wants to join a group, it will start the joining process immediately, and the nodes can join the multicast group very quickly as shown in Fig. 5(d). SPBM is seen to have the largest joining delay most of the time. As described in Section II, with the use of periodic levelby-level membership update, it may take a long time for a bottom level square of SPBM to disseminate its membership change information to the upmost level. In ODMRP, the mesh structure is built on the demand of the source, and the source sends out a JOIN QUERY periodically to refresh the mesh structure. If the nodes want to join a group, they need to wait until the next mesh refreshing period. The refreshing interval is set as 3 seconds according to [15]. From the figure, the average joining delay of ODMRP decreases with the increase of mobility, as the higher moving speed helps a member connect to the source more quickly in the non-geographic mesh structure.

In summary, compared to ODMRP and SPBM, RSGM provides much more reliable transmissions in a dynamic environment with the support of its virtual-zone based membership management and stateless virtual delivery trees. At the highest mobility, it achieves $40 \%$ and $15 \%$ higher delivery ratios than ODMRP and SPBM, respectively. It also has the minimum control overhead and group joining delay under all the mobility. The control overhead of ODMRP and RSGM are comparable, while the overhead of SPBM is about six times their overhead. Similarly, the joining delay of SPBM is also six times that of RSGM. The joining delay of ODMRP reduces with the increase of mobility, and is still three times that of RSGM at the highest mobility. The increase of mobility also leads to the increase of path lengths of the two geometric multicast protocols, which is partially due to the transmission inefficiency of the underlying geometric unicast protocol and partially due to the increased failures of the longer-path transmissions in ODMRP.

3) Impact of node density: Since geographic routing is sensitive to the node density and performs better in a dense network, we also study the impact of node density on the performance.

As expected, both RSGM and SPBM have higher delivery ratios at higher node density (Fig. 6 (a)). All protocols have low packet delivery ratios in a sparse network where the network graph is weakly connected. RSGM keeps a higher delivery ratio and its delivery ratio increases faster as the network density increases. According to the feature of geographic routing, when the node density is smaller, there is a smaller chance for an intermediate node to find a neighbor closer to the destination, and the recovery forwarding has to be used more frequently which introduces longer paths for control messages and data packets. As a result, RSGM and SPBM have higher control overheads and longer end-to-end paths in a sparser network as shown in Fig. 6(b)(c). SPBM's control overhead increases quickly when the node density becomes higher as more nodes are involved in its periodic multi-level flooding for membership management.

For all the protocols, the disconnected topology graph in a sparse network leads to a longer joining delay (Fig. 6(d)). The followed slight increase of the joining delay at high node density is due to more transmission collisions. Such increase is more obvious for SPBM since its higher control overhead results in more collisions.

Overall, all the protocols perform better in a denser network, and RSGM has consistently higher delivery ratio than SPBM and ODMRP. SPBM has a significantly higher control overhead and joining delay in a dense network as a result of its periodic multi-level flooding of membership management message, while RSGM remains to have the lowest delay as it allows group members to join and leave the group immediately on demand. The geometric protocols have longer transmission paths in a sparse network due to the more frequent use of recovery forwarding of the underlying geometric unicast protocol.

4) Impact of group size: Next we demonstrate the protocol performance with different group sizes from 10 members to 200 members.

Fig. 7 shows that RSGM is scalable to group size and performs better than ODMRP and SPBM with various group 
(a)

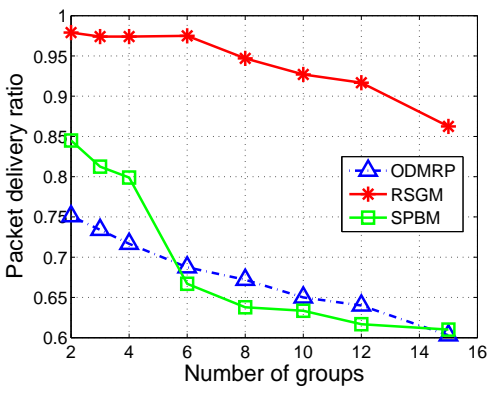

(c)

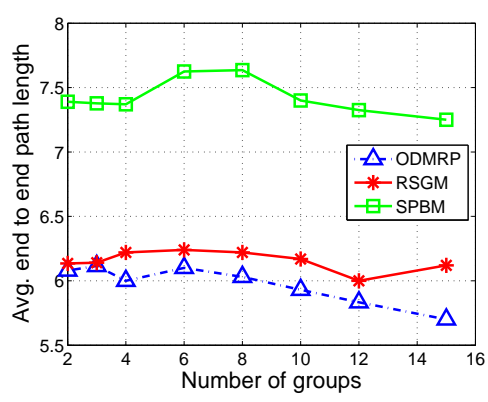

(b)

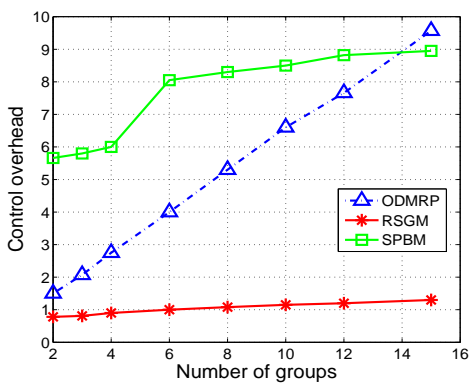

(d)

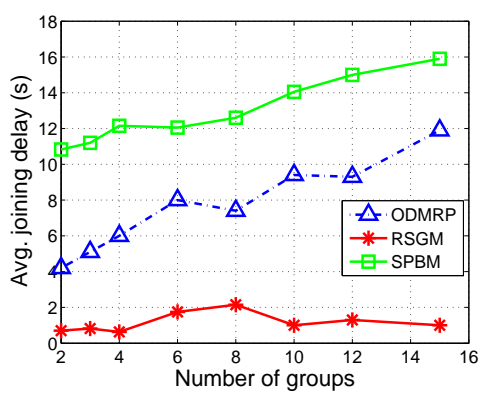

Fig. 8. Performance vs. number of groups (1 source per group, totally 120 group members): (a) packet delivery ratio; (b) normalized control overhead; (c) average path length; (d) average joining delay.

(a)
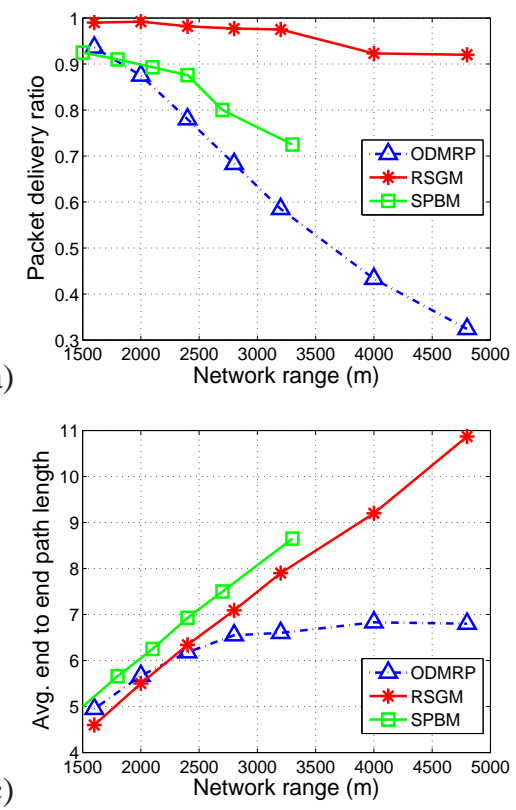

(b)

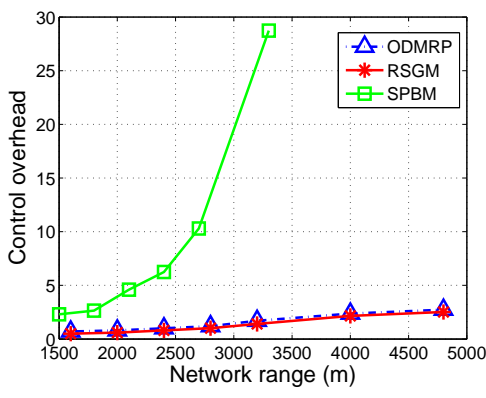

(d)

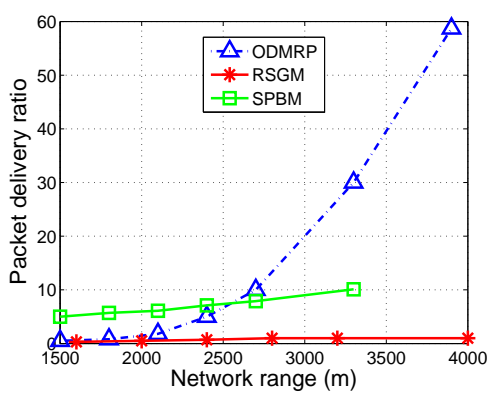

Fig. 9. Performance vs. network size (1 group, 1 source, 100 group members): (a) packet delivery ratio; (b) normalized control overhead; (c) average path length; (d) average joining delay.

sizes. The delivery ratio of RSGM remains higher than $98 \%$ under all group sizes. When the group size increases, ODMRP and SPBM make more successful deliveries. The mesh structure in ODMRP has more redundancy when more nodes join the multicast group and will provide more robust delivery paths. The membership of the squares in SPBM becomes more stable with a larger group size.

In Fig. 7(b), ODMRP and SPBM are seen to have high control overhead when the group size is small. In ODMRP, all the mobile nodes are involved in the periodic flooding of JOIN QUERY, which results in a higher normalized control overhead. In SPBM, the proactive multi-level control message flooding causes much more unnecessary overhead when the group size is small relative to the total number of mobile nodes. While in RSGM, a smaller group size will lead to fewer member zones and hence fewer LEADER and REPORT messages, and most of the control overhead is caused by the proactive beacons sent by each node in the underlying geographic unicast routing protocol. When the group size increases, the normalized control overhead of all the protocols reduces accordingly with more data packets delivered. With more distant members joining the group, the average path 
lengths become longer for all the protocols (Fig. 7 (c)).

The change of group size has different impacts on the joining delay of the three protocols as shown in Fig. 7 (d). In RSGM, as the group size increases, there are more member zones, hence more joining processes are completed inside local zones, which results in a decrease of joining delay for RSGM. The joining delay of SPBM drops as group size goes up, because the memberships of the squares become more stable when the group size is larger and the joining process of a node triggers fewer levels of membership changes in the quad-tree. Relying on the periodic JOIN QUERY message to refresh the mesh structure for node to join a group, the group size does not have a significant impact on the joining delay of ODMRP.

In summary, RSGM has more than $98 \%$ delivery ratios for all the group sizes, and it does not incur unnecessary control overhead when there is no member in a zone. In contrast, there are higher control overheads for ODMRP and SPBM due to their uses of periodic flooding messages regardless of the group size, which result in their higher normalized overheads at a smaller group size. RSGM has comparable path length to ODMRP, while SPBM has the longest path lengths under all the group sizes. The group size has little impact on the joining delay of RSGM, while SPBM has a significantly higher joining delay when the network is sparse.

5) Impact of the number of groups: To study the impact of the number of groups, we conducted simulations with 2, 3, 4, $6,8,10,12$ and 15 groups. The total number of members are fixed as 120 , thus for each scenario, there are respectively 60 , $40,30,20,15,12,10$ and 8 members per group.

The simulation results in Fig. 8 indicate that RSGM is also scalable to the number of groups. RSGM outperforms ODMRP and SPBM under different numbers of groups for both delivery ratio and control overhead. The delivery ratios of all protocols drop when the number of groups increases because of the heavier transmission load, which results in more collisions and hence more packet loss. SPBM is shown to have the sharpest decrease in delivery ratio since its higher control overhead and data packet transmission overhead (Fig. 8 (b)(c)) lead to more collisions. Also, according to the settings, the group size is smaller with more groups, so SPBM has less stable square membership. From Fig. 8 (b), ODMRP's control overhead increases almost linearly with the number of groups, as more control overhead is generated when more sources periodically flood JOIN QUERY. When the number of groups increases, the smaller group size and heavier transmission load also lead to the increase of joining delays for ODMRP and SPBM as seen in Fig. 8 (d), while the joining delay of RSGM is little impacted.

In summary, RSGM scales well with the number of groups, and has consistently higher delivery ratio than those of ODMRP and SPBM, and achieves more than 40\% higher delivery ratio than both peer protocols at the highest number of groups tested. Its control overhead, average path length, and joining delay are little impacted by the number of groups, while the control overhead of ODMRP increases almost linearly with the number of groups as a result of the periodic flooding of JOIN QUERY by a source.
6) Impact of network size: To study the protocol's scalability to network size, we varied the network-range from $1500 m \times 1500 m$ to $4800 m \times 4800 m$. The node density is kept as before, thus the total number of nodes is varied from 156 nodes to 1600 nodes. Since the periodic local and networkwide message flooding in SPBM saturates the machine's memory faster, we run simulations on SPBM with the network size increased up to only $3300 m \times 3300 m$ with 756 nodes.

RSGM has a much better scalability to network size than ODMRP and SPBM as demonstrated in Fig. 9. The delivery ratios of both ODMRP and SPBM drop quickly with the increase of network size, while the delivery ratio of RSGM remains at above $90 \%$ at the largest network size. The periodic flooding-based mechanism of ODMRP makes it hard to scale to a large network size, as the broadcasting is unreliable and also causes more transmission collisions. When the network size reaches $4800 m \times 4800 m$ with 1600 nodes, the delivery ratio of RSGM is three times that of ODMRP, while the RSGM has more than $40 \%$ higher delivery ratio than that of SPBM at the network size of $3300 m \times 3300 m$, beyond which SPBM simulation could not be run. The control overheads of all the protocols increase as the network size increases. For ODMRP, more nodes are involved in the periodic JOIN QUERY flooding. For RSGM, a larger network range leads to a longer forwarding path for network-tier control messages. The underneath geographic unicast protocol also generates more beacons with more nodes. The periodic multi-level message flooding in SPBM causes its control overhead to increase much more sharply than those of RSGM and ODMRP. As expected, the path lengths of all the protocols increase when the network range is enlarged as shown in Fig. 9 (c). ODMRP has a shorter delivery path in a large network, because its unreliable broadcast-based forwarding makes data packets hard to reach farther away nodes and more packets with long paths are dropped in ODMRP.

All three protocols also have longer joining delay when the network size increases as in Fig. 9 (d). The joining delay of ODMRP is significantly impacted by the network size, as both its periodic network-wide flooding of JOIN QUERY and its broadcast-based packet forwarding do not perform well at a large network size. More data collisions during the flooding will result in a longer waiting time for a group member to receive the first data packet from the source, and a larger number of packet loss as confirmed by the low delivery ratio in Fig. 9 (a). For SPBM, with the increase of the number of the quad-tree levels, the membership change of a node may need to go through more levels to reach the source leading to a longer joining delay. The joining delay of RSGM only rises slightly at a large network size, because a newly joined zone may be farther away from the source.

In summary, RSGM can perform much better than SPBM and ODMRP in a large network, and has a significantly higher delivery ratio, lower control overhead, and lower joining delay due to its virtual and reliable membership management and transmission infrastructures. 


\section{DISCUSSIONS}

In this section, we further discuss the issues of handling multiple sources per group and multiple clusters per zone.

In the case that a group $G$ has multiple sources, one of them (e.g., the one with the smallest address) can be chosen as a representative for the sources (denoted by RS). The zone leaders send the periodic REPORTs only to RS. Only when the zone list for $\mathrm{G}$ maintained by RS changes, i.e., some zone records are removed due to timeout, it notifies other sources through a MAP message. The MAP message carries a bit map with each bit corresponding to a zone in the network, and the bit is set to one for a member zone and zero otherwise. As presented in Section III-B2, besides the periodic reporting, a zone leader also needs to send a REPORT when the membership of the zone changes. In the second case, the REPORT needs to be sent to all the sources instead of only RS so that the sources can start or terminate sending multicast packets to the zone in time.

The nodes in a zone may form multiple clusters, which are not connected in the zone but are connected on the network topology graph through some nodes outside the zone. In this case, two nodes in different clusters can communicate with each other through unicast, but an intra-zone flooding message initiated in one cluster may not reach other clusters. Many protocols use a smaller zone size so that all nodes in a zone are within each others' transmission range, however, this still cannot solve the problem if some obstacles in the zone (e.g., a hill or a building) block the radio communications. In RSGM, when there are multiple clusters in a zone, without knowing the existence of other clusters, each cluster containing member nodes will elect a leader. From the zone ID, S can detect the existence of multiple clusters in a zone and sends data packets to each of the cluster. Some clusters may merge later and a new leader will be elected. For the Source Home, similar to a member zone, the zone leader needs to report to $S$ periodically. On detecting multiple clusters in the Source Home, S informs the status to the cluster leaders. When a REGISTER sent from $S$ reaches a cluster, the cluster leader will unicast a copy of the message to other cluster leaders, which will flood the message within their own clusters. For efficiency, the source can designate a new Source Home.

\section{CONCLUSIONS}

In this paper, we have designed a robust and scalable geographic multicast protocol RSGM for MANET. In RSGM, stateless virtual transmission structures are used for simple management and robust forwarding. Both data packets and control messages are transmitted along efficient tree-like paths without the need of explicitly creating and maintaining a tree structure. Scalable membership management is achieved through a virtual-zone-based two-tier infrastructure. A Source Home is defined to track the locations and addresses of the multicast sources to avoid the periodic network-wide flooding of source information, and the location service for group members is combined with the membership management to avoid the use of an outside location server. The position information is used in RSGM to guide the zone structure building, membership management and packet forwarding, which reduces the maintenance overhead and leads to more robust multicast forwarding when the topology changes. We have also handled the empty zone problem which is challenging for the zone-based protocols.

We quantitatively analyze the control overhead of the proposed RSGM protocol and our analysis results indicate that the per-node cost of RSGM keeps relatively constant with respect to network size and group size. We have performed extensive simulations to evaluate the performance of RSGM. Our results demonstrate that RSGM not only outperforms the existing geographic multicast protocol SPBM and widely-used multicast protocol ODMRP but can also scale to a large group size, large number of groups, and large network size. To be more specific, RSGM has much higher packet delivery ratio than SPBM and ODMRP under different moving speeds, node densities, group sizes, number of groups, and network sizes. The difference becomes more evident as the moving speed increases. For example, at the speed of $40 \mathrm{~m} / \mathrm{s}$, the delivery ratio of RSGM is about $20 \%$ higher than that of SPBM, and $40 \%$ higher than that of ODMRP.

RSGM scales well with the group size, and achieves more than $98 \%$ delivery ratio under all the group sizes studied. On the other hand, the delivery ratios of SPBM and ODMRP drop significantly when there is a large number of groups in the network or when the network size is large. For example, when there are 15 groups, the delivery ratio of RSGM is more than $40 \%$ higher than that of SPBM and ODMRP. For a network size of $3300 \mathrm{~m} \times 3300 \mathrm{~m}$, the delivery ratio of RSGM is about $40 \%$ larger than that of SPBM, and $90 \%$ higher than that of ODMRP, and the delivery ratio of RSGM triples that of ODMRP when the network size reaches $4800 \mathrm{~m}$ x $4800 \mathrm{~m}$. In almost all the simulation scenarios, RSGM has the lowest control overhead with the support of virtual-zonebased hierarchical membership management, virtual trees for message transmissions, and Source Home for source tracking. Due to the use of hierarchical structure, the average endto-end path lengths of both SPBM and RSGM are higher than that of ODMRP, with the path length of SBMP much longer than RSGM. The shorter path length of ODMRP is also due to the higher dropping probability a packet experiences when passing though a longer path. Our studies indicate that geometric information and virtual infrastructures can be used together to achieve much more reliable and scalable multicast packet delivery in the presence of constant topology change of MANET.

\section{ACKNOWLEDGEMENTS}

Several people have been instrumental in allowing this paper to be completed. We would like to thank especially Shan Chu, who helped generate the figures of the paper, and the authors of SPBM, who generously shared their simulation codes with us. Xin Wang's research was supported by US NSF under grant numbers CNS 0751121 and CNS 0628093. Yuanyuan Yang's research was supported by US NSF under grant numbers ECCS-0801438 and ECS-0427345 and US ARO under grant number W911NF-09-1-0154. 


\section{REFERENCES}

[1] W. Wu , J. Cao , J. Yang and M. Raynal. Design and Performance Evaluation of Efficient Consensus Protocols for Mobile Ad Hoc Networks. IEEE Transactions on Computers, Vol. 56, No. 8, Aug. 2007. pp. 1055 $-1070$.

[2] R. Beraldi and R. Baldoni. A Caching Scheme for Routing in Mobile Ad Hoc Networks and Its Application to ZRP. IEEE Transactions on Computers, Vol. 52, No. 8, Aug. 2003. pp. 1051 - 1062.

[3] Y. C. Tseng , S. Y. Ni and E. Y. Shih. Adaptive Approaches to Relieving Broadcast Storms in a Wireless Multihop Mobile Ad Hoc Network. IEEE Transactions on Computers, Vol. 52, No. 5, May 2003. pp. 545 557.

[4] C.-C. Chiang, M. Gerla and L. Zhang. Forwarding group multicast protocol (FGMP) for multihop, mobile wireless networks. AJ. Cluster Comp, Special Issue on Mobile Computing, 1(2):187-196, 1998.

[5] S. Wu and K.S. Candan. GMP: Distributed Geographic Multicast Routing in Wireless Sensor Networks. In Proc. 26th IEEE Intl Conf. Distributed Computing Systems (ICDCS 06), 2006.

[6] S.M.Das, H. Pucha and Y.C. Hu. Distributed Hashing for Scalable Multicast in Wireless Ad Hoc Network. In IEEE Transactions on Parallel and Distributed Systems (TPDS), Vol. 19(3), March 2008.

[7] J. Yoon, M. Liu and B. Noble. Random Waypoint Considered Harmful. Proc. IEEE INFOCOM 03, 2(4), Apr. 2003.

[8] L. Ji and M. S. Corson. Differential destination multicast: a MANET multicast routing protocol for small groups. In Proc. IEEE Infocom01, Anchorage, Alaska, April 2001.

[9] I. Abraham, D. Dolev and D. Malkhi. LLS: a locality aware location service for mobile ad hoc networks. In Workshop on Discrete Algorithms and Methods for MOBILE Computing and Communications (DialM), 2004.

[10] J. Li, J. Jannotti, D. S. J. D. Couto, D. R. Karger and R. Morris A scalable location service for geographic ad hoc routing. In MOBICOM, pp. 120-130, 2000.

[11] J. J. Garcia-Luna-Aceves and E. Madruga. The core-assisted mesh protocol. IEEE JSAC, pp. 1380-1394, August 1999.

[12] M. Gerla, S. J. Lee and W. Su. On-demand multicast routing protocol (ODMRP) for ad hoc networks. Internet draft, draft-ietf-manet-odmrp02.txt, 2000

[13] L. Ji and M.S. Corson. A lightweight adaptive multicast algorithm. In GLOBECOM, pp. 1036-1042, 1998.

[14] B. Karp and H. T. Kung. Greedy perimeter stateless routing for wireless networks. In MOBICOM, pp. 243-254, August 2000.

[15] S. Lee, W. Su, J. Hsu, M. Gerla and R. Bagrodia. A performance comparison study of ad hoc wireless multicast protocols. In IEEE INFOCOM, 2000.

[16] S. Basagni, I. Chlamtac and V.R. Syrotiuk. Location aware, dependable multicast for mobile ad hoc networks. Computer Networks, 36(5-6):659670, August 2001

[17] K. Chen and K. Nahrstedt. Effective location-guided tree construction algorithms for small group multicast in MANET. In IEEE INFOCOM, pp. 1180-1189, 2002.

[18] M. Mauve, H. Fubler, J. Widmer and T. Lang. Position-based multicast routing for mobile ad-hoc networks. Poster section in ACM MOBIHOC June 2003.

[19] E.M. Royer and C.E. Perkins. Multicast operation of the ad hoc ondemand distance vector routing protocol. In MOBICOM, pages 207-218, August 1999.

[20] M. Transier, H. Fubler, J. Widmer, M. Mauve and W. Effelsberg. Scalable position-based multicast for mobile ad-hoc networks. In Proc of the First International Workshop on Broadband Wireless Multimedia: Algorithms, Architectures and Applications (BroadWim 2004), San Jose, CA, October 2004.

[21] M. Transier, H. Fubler, J. Widmer, M. Mauve and W. Effelsberg. A hierarchical approach to position-based multicast for mobile adhoc networks. Technical Report TR-04-002, Department of Computer Science, University of Mannheim, Germany, January 2004.

[22] UCLA Parallel Computing Laboratory. http://pcl.cs.ucla.edu/projects/glomosim/.

[23] S-C. M. Woo and S. Singh. Scalable routing protocol for ad hoc networks. Wireless Networks, vol 7, pp. 513-529, 2001.

[24] C. Wu, Y. Tay and C.-K. Toh. Ad hoc multicast routing protocol utilizing increasing id-numbers (AMRIS) functional specification. Internet draft, November 1998

[25] X. Zhang and L. Jacob. Multicast zone routing protocol in mobile ad hoc wireless networks. in Proceedings of Local Computer Networks, 2003 (LCN 03), October 2003.
[26] X. Xiang, Z. Zhou and X. Wang. Self-adaptive on demand geographic routing protocols for mobile ad hoc networks. In IEEE INFOCOM Minisymposium, Anchorage, Alaska, May 2007.

[27] C. Gui and P. Mohapatra. Scalable Multicasting for Mobile Ad Hoc Networks. In Proc. IEEE INFOCOM, Mar. 2004.

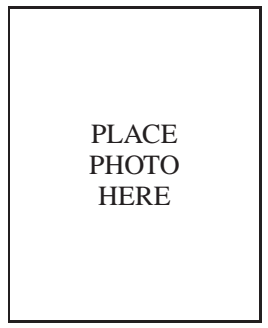

Xiaojing Xiang (M'06) received her BS and MS degrees in computer science from Nanjing University, Nanjing, China, and her $\mathrm{PhD}$ degree in computer science and engineering from the State University of New York at Buffalo, Buffalo, New York.

She is currently with Microsoft Corporation, Redmond, Washington. Her research interests include protocol design and analysis in mobile ad hoc networks, architecture design for service provisioning, routing and cross-layer protocol design in computer networks, pervasive computing and communications, as well as next generation Internet technologies.

\begin{tabular}{|c|}
\hline \\
\\
PLACE \\
PHOTO \\
HERE \\
\end{tabular}

Xin Wang (M'01 / ACM'04) received her BS and MS degrees in telecommunications engineering and wireless communications engineering from Beijing University of Posts and Telecommunications, Beijing, China, and her $\mathrm{PhD}$ degree in electrical and computer engineering from Columbia University, New York, NY.

She is currently an assistant professor in the department of Electrical and Computer Engineering of the State University of New York at Stony Brook, Stony Brook, New York. Before joining Stony Brook University, she was a Member of Technical Staff in the area of mobile and wireless networking at Bell Labs Research, Lucent Technologies, New Jersey and an assistant professor in the department of Computer Science and Engineering of the State University of New York at Buffalo, Buffalo, New York. Her research interests include analysis and architecture design in wireless networks and communications, mobile and distributed computing, infrastructure design and performance enhancement across network layers, applications and heterogeneous networks, network and mobility management, QoS, signaling and control, as well as support for advanced network services and applications.

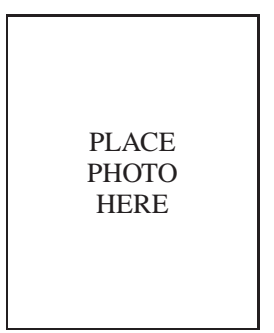

Yuanyuan Yang (M'91/SM'98/F'09) received the BEng and MS degrees in computer science and engineering from Tsinghua University, Beijing, China and the MSE and PhD degrees in computer science from Johns Hopkins University, Baltimore, Maryland. She is a Professor of Computer Engineering and Computer Science at Stony Brook University, New York, and the Director of Communications and Devices Division at New York State Center of Excellence in Wireless and Information Technology (CEWIT). Dr. Yang's research interests include wireless networks, optical networks, high speed networks, and parallel and distributed computing systems. Her research has been supported by the National Science Foundation (NSF) and U.S. Army Research Office (ARO). Dr. Yang has published over 200 papers in major journals and refereed conference proceedings and holds six U.S. patents in these areas. She is currently an Associate Editor for IEEE Transactions on Computers and a Subject Area Editor for Journal of Parallel and Distributed Computing. She has served as an Associate Editor for IEEE Transactions on Parallel and Distributed Systems. She has served as a general chair, program chair or vice chair for several major conferences and a program committee member for numerous conferences. She is a Fellow of the IEEE. More information about her and her research can be found at http://www.ece.sunysb.edu/ yang. 